\title{
АССОЦИАЦИЯ ЦИРКАДИАННОЙ ДИЗРИТМИИ С ВОЗНИКНОВЕНИЕМ МЕТАБОЛИЧЕСКИХ РАССТРОЙСТВ
}

\section{ASSOCIATION OF CIRCADIAN DYSRHYTHMIA WITH THE GENESIS OF METABOLIC DISORDERS}

\section{O. Vengrzhinovskaya M. Fadeeva}

Summary. The functioning of the human body is subject to certain chronobiological laws. Circadian (circadian, circadian) rhythms are autonomous from external influences and last from 20 to 28 hours, but normally they are synchronized daily with the 24-hour day-night cycle. The generation of circadian rhythms is carried out by the suprachiasmal nuclei of the anterior hypothalamus. Melatonin, the main hormone of the pineal gland, is an important pacemaker that transmits information about the light regime to the body, regulating the sleepwake cycle. The functioning of the suprachiasmatic nuclei and pineal gland is in a reciprocal relationship. The level of activity of metabolic and physiological processes, the secretion of most hormones and biologically active substances are under biorhythmological control and have a certain seasonal and daily (in daily 24-hour cycles) dynamics, and in some cases are closely related to sleep (certain stages). [1] This organization is essential to increase the metabolic efficiency of the body. Circadian mismatch, most often caused by untimely exposure to light, disturbed sleep or food intake, impairs glycemic and lipid control, negatively affects factors involved in energy balance and body weight control, disorders the rhythms of hormone secretion, which contributes to the appearance of metabolic and hormonal disorders. In patients with obstructive sleep apnea syndrome, circadian rhythm disorders occur regardless of external factors. This article discusses the influence of the circadian metabolic system: regulation of glucose and lipid metabolism, the organization of the correct secretion of hormones, the secretion of adipocytokines, as well as the consequences of circadian dysrhythmia in obese patients. This knowledge will help to optimize the management of such patients in the practice of an endocrinologist.

Keywords: somnology, circadian rhythms, circadian disorders, endocrinology, obesity, metabolic syndrome, carbohydrate metabolism, lipid metabolism.
Венгржиновская Оксана Игоревна

Врач-ординатор эндокринолог, ФГБУ

«Национальный медицинский исследовательский иентр эндокринологии» Министерства здравоохранения Российской Федерации, Москва vengrzhinovskay@gmail.com

Фадеева Мария Игоревна

Врач-эндокринолог, ФГБУ «Начиональный медицинский исследовательский чентр эндокринологии» Министерства здравоохранения Российской Федерачии, Москва

Аннотачия. Функциональность человеческого организма подчиняется определенным хронобиологическим закономерностям. Циркадианные (циркадные, околосуточные) ритмы являются автономными от внешних воздействий и имеют длительность от 20 до 28 ч, однако в норме ежедневно синхронизированы с 24-часовым циклом «день-ночь». Генерацию циркадианных ритмов осуществляют супрахиазмальные ядра переднего гипоталамуса. Мелатонин — главный гормон эпифиза — является важным пейсмейкером, передающим информацию о световом режиме организму, регулируя цикл «сон-бодрствование». Функционирование супрахиазмальных ядер и эпифиза находится в реципрокных отношениях. Уровень активности метаболических и физиологических процессов, секреция большинства гормонов и биологически активных веществ находятся под биоритмологическим контролем и имеют определенную сезонную и суточную (в ежедневных 24-часовых циклах) динамику, и в ряде случаев тесно связаны со сном (определенными его стадиями).[1] Такая организация является необходимой для повышения метаболической эффективности организма. Циркадианное рассогласование, наиболее часто вызванное несвоевременным воздействием света, нарушением режима сна или приема пищи, ухудшает гликемический и липидный контроль, отрицательно влияет на факторы, участвующие в энергетическом балансе и контроле массы тела, нарушает ритмы секреции гормонов, что способствует появлению метаболических и гормональных расстройств. В данной статье рассмотрено влияние циркадианной системы на метаболизм: регуляцию обмена глюкозы и липидов, организацию правильной секреции гормонов, секрецию адипоцитокинов, а также последствия циркадианной дизритмии у больных ожирением. Эти знания помогут оптимизировать ведение таких больных в практике врача-эндокринолога.

Ключевые слова: сомнология, циркадные ритмы, циркадианнаые нарушения, эндокринология, ожирение, метаболический синдром, углеводный обмен, липидный обмен. 


\section{Ввемение}

«C ircadian» происходит от латинских корней «circa», означающего «вокруг», и «diēm», означающего «день». Циркадианные ритмы - это периодические паттерны, которые повторяются примерно каждые 24 часа [2], и генерируются внутри организма (эндогенно) даже при отсутствии света или других внешних воздействий.

Циркадианный ритмы крайне важны, поскольку они неразрывно связаны с различными гормональными и метаболическими процессами, они играют важную роль в поддержании гомеостаза и секреции гормонов в ежедневном (суточном) цикле.

Для оптимизации метаболической эффективности организма, циркадианные ритмы разделяют во времени противоположные метаболические процессы анаболизм и катаболизм (рис. 1). [3]

Циркадианная система включает в себя центральный осциллятор, расположенный в билатерально симметричных супрахиазмальных ядрах (СХЯ) переднего гипоталамуса в головном мозге и генерирующий эндогенную продолжительность циркадианного ритма, и ряд вторичных осцилляторов в периферических тканях и органах, разбросанных по всему телу, включая печень, поджелудочную железу, желудочно-кишечный тракт, скелетные мышцы, жировую ткань, сердце и др. (рис. 2).

Молекулярной основой циркадианных часов является внутриклеточная транскрипционно-трансляционная саморегулирующаяся петля обратной связи: белки - продукты группы часовых генов (включая CLOCK, BMAL1, PER 1/2, CRY1/2 и REV-ERB-a), которые кодируют факторы транскрипции, чтобы произвести цикл с периодом около 24 часов [4].

Работа всей системы часов модулирует широкий спектр метаболических мишеней, таких как глюкокортикоиды, моллекулы АМФ (аденозин-монофосфата), активированную протеинкиназу (adenosine monophosphate-activated protein kinase, AMPK), синтез жирных кислот и холестерина и печеночный транскрипционный фактор(сAMP response element-binding protein (CREB) для модуляции глюконеогенеза [5]. В совокупности целый ряд метаболических процессов (включая чувствительность к инсулину, секрецию инсулина, синтез холестерина, окисление жира и расход энергии) и секреция гормонов и многих биоактивных веществ следуют ритму в течение 24-часового дня.

Ритм центральных биологических часов в первую очередь регулируется первичным таймером (zeitgeber) - дневным светом, действующим через фоторецепторы сетчатки и ретино-гипоталамический тракт, необходимым для ежедневной смены фаз. Периферические ткани интегрируют сигналы от центрального осциллятора с факторами окружающей среды и поведенческими факторами (включая свет, сон, физическую активность, питание)а также со своими собственными автономными ритмами для циркадианной синхронизации метаболизма. Совместно с СХЯ гипоталамуса определенную роль в циркадианной регуляции в организме играет гормон мелатонин, гамма-аминомасляная кислота (ГАМК), внешние факторы (свет, режим работы, время приема пищи).

\section{ЦиркаАианная Аизритмия}

Рассогласование внутренних часов организма с окружающим ритмом жизни может быть вызвано, например, длительным перелетом со сменой часовых поясов (так называемый джетлаг) или наиболее часто встречается социальный джетлаг - из-за сменного режима работы, переработки на дому, связанные с работой или учебой в институте/школе, нарушение ритма сна/бодрствования из-за длительного отдыха. Социальный джетлаг ассоциирован с более высоким индексом массы тела (ИМТ) и риском перехода от избыточного веса к ожирению, [6] повышением риска развития сахарного диабета 2 типа (СД 2) и метаболического синдрома (МС). Более поздний хронотип также ассоциируется с риском развития СД 2 и более высоким уровнем гликированного гемоглобина (НbA1c) при СД 2 и предиабете [7].

Таким образом, нарушение циркадианных ритмов увеличивает риск развития метаболических расстройств и нарушений эндокринного гомеостаза, что объясняет столь драматический рост ожирения и СД 2, поскольку такие факторы как переедание или гиподинамия уже не могут в полной мере сделать этого. Нарушенный паттерн сна/бодрствования приводит к изменению молекулярных механизмов внутриклеточных циркадианных ритмов (концентрации глюкозы, свободных жирных кислот (СЖК), триглицеридов (ТГ), гормонов, например, инсулина, глюкокортикостероидов (ГКС)).

\section{ЦиркаАианные ритмы метаболизма глюкозы}

Множество исследований подтвердили существование суточного ритма толерантности к глюкозе в ходе перорального ее приема. При этом величина суточных вариаций толерантности к глюкозе поразительно велика: взрослые с нормальной толерантностью к глюкозе утром могут быть метаболически эквивалентны предиабетическому состоянию вечером. 


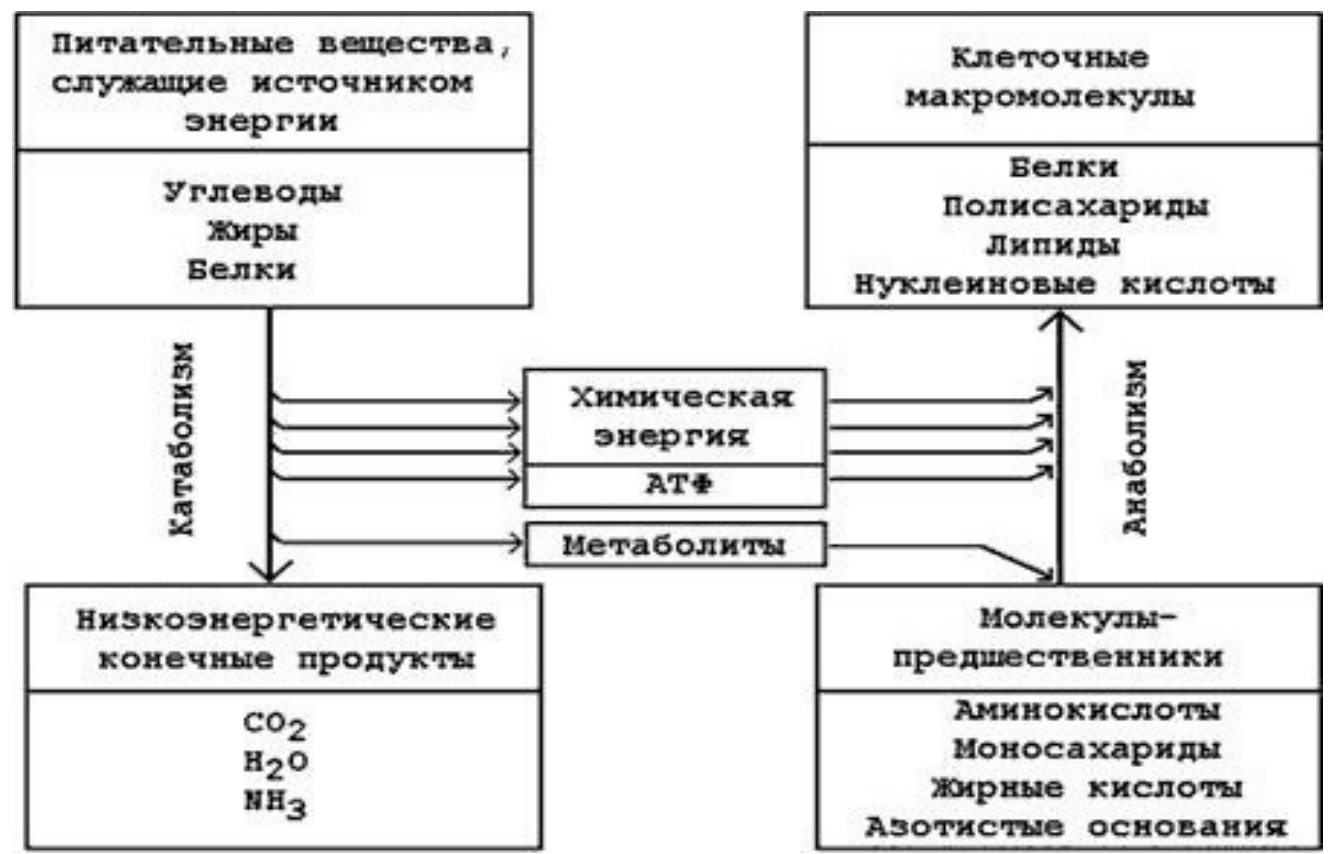

Pис. 1

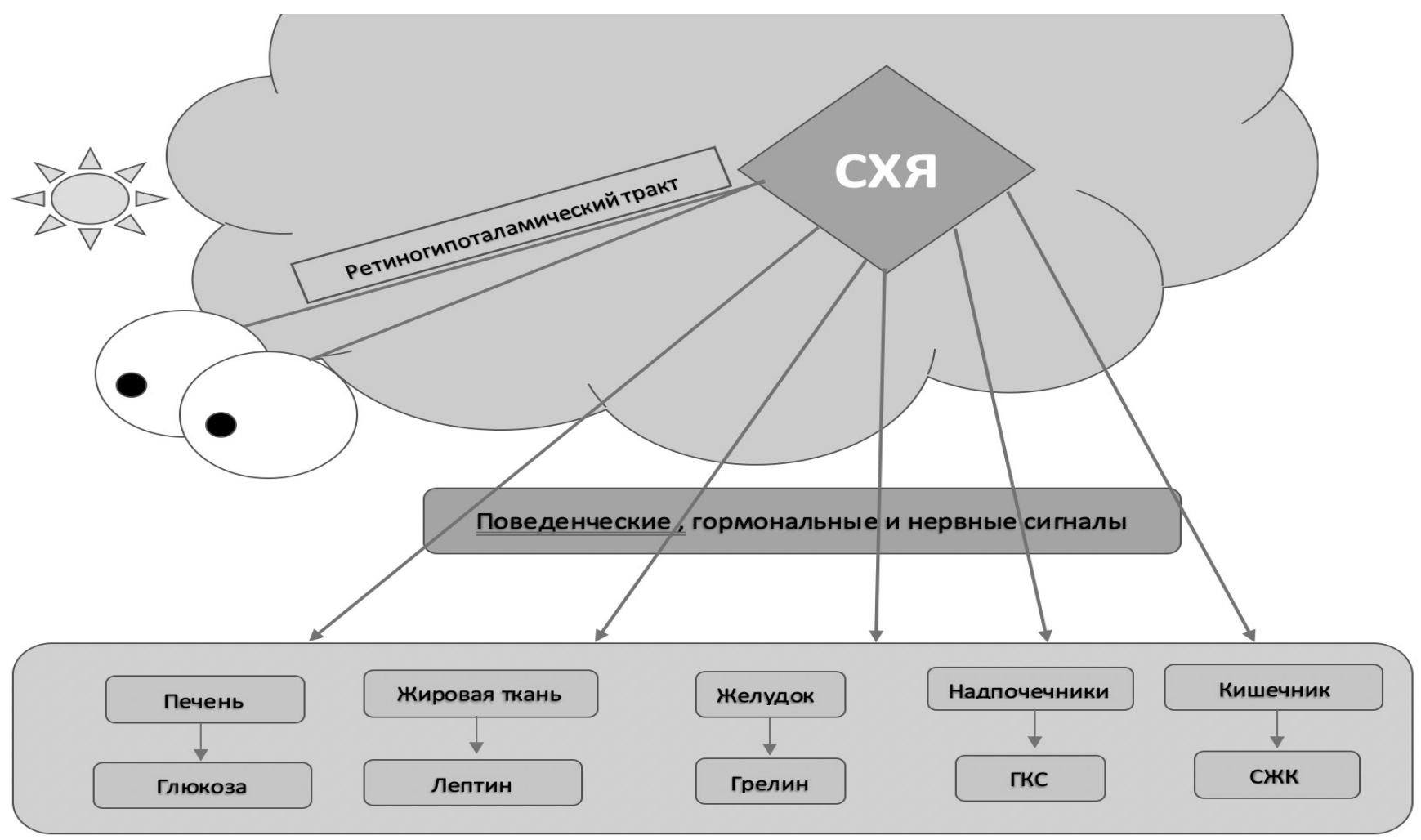

Схя -супрахиазмальное ядро гипоталамуса

ГКС- глюкокортикострероиды

СЖк свобожные жирные кислоты

Рис. 2 
Такие суточные колебания толерантности к глюкозе могут быть связаны: с суточными ритмами чувствительности ß-клеток поджелудочной железы (ПЖ); с суточными ритмами секреции инсулина и его клиренса; ритмами периферической чувствительности к инсулину. Так, чувствительность, Љ-клеток ПЖ, измеряемая по толерантности к глюкозе, выше утром, чем в другое время суток [8]. Большинство исследований показывает, что клеточные автономные циркадианные часы в ß-клетках ПЖ являются ключевым регулятором гомеостаза глюкозы млекопитающих и человека, и что их дисрегуляция тесно связана с метаболическими расстройствами у человека. При наличии циркадианного сдвига (сон с 9:00 до 14:00) происходит достоверное снижение чувствительности к инсулину (с 58\% до 32\%) при сопоставимых длительности сна, калоражу пищи, продолжительности фаз сна в сравнении со здоровыми испытуемыми без циркадианного сдвига сна (сон с 00:30 до 05:30). [9].

Нарушение суточных ритмов толерантности к глюкозе, секреции инсулина и периферической чувствительности к нему ослабляются либо замедляются у лиц с ожирением. То же самое отмечается при предиабете, а у взрослых с СД 2 они отсутствуют или инвертированы (фаза задерживается на несколько часов). В исследовании с постоянной инфузией глюкозы обнаружено, что ритм толерантности к глюкозе был изменен у лиц с ожирением, их ритмы утилизации глюкозы и секреции инсулина были снижены [10].

Таким образом нарешение суточного ритма метаболизма глюкозы может привести к метаболическому синдрому, развитию преддиабета или СД 2типа.

\section{Циркалианные ритмы}

обмена $\wedge$ ии $\triangle \mathrm{OB}$

Секреция большинства липидов в отличие от других метаболитов плазмы в большей степени регулируется циркадианной системой. Пик синтеза холестерина (XC) также подчиняется циркадианному ритму разнятся. Большинство исследований показывают, что пик приходится на 22.00 ч. Пик выработки триглицеридов (ТГ), зависит от времени приема пищи. Он отмечается после обеда, причем у мужчин после приема пищи наблюдается в 2 раза более высокое максимальное повышение их уровня в сыворотке крови, чем у женщин, и более высокая суточная вариабельность (36\% у мужчин против 24\% у женщин) [11]. Эти различия могут быть обусловлены влиянием эстрогенов на липидный обмен, возраста и метаболического фенотипа.

Суточный ритм также имеется у ацилкарнитинов, которые участвуют в окислении жирных кислот, перенося их из цитоплазмы в митохондрии. В одном из исследований при биопсии мышц у 13 здоровых женщин с избыточной массой тела показали, что гены, регулирующие окисление жирных кислот, подавлены на 38-82\% в вечернее время по сравнению с утренним, в то время как гены, вовлеченные в липогенез de novo, активированы на 51-87\%. Т.е. экспрессия генов, вне зависимости от соответствующих изменений липолиза в жировой ткани, благоприятствует сдвигу в разделении жирных кислот в скелетной мышце от окисления утром до липогенеза вечером, что возможно обуславливает развитие ожирения при синдроме «ночной еды». [12]

Многие анаболические ритмы у людей достигают пика в биологическое утро или ранним днем. Прием пищи в соответствии с этими ритмами путем его переноса на более раннее время в дневное время, способствует снижению массы тела у взрослых. Эти преимущества могут быть обусловлены главным образом циркадианными изменениями в постпрандиальных метаболических путях, в особенности в периферических тканях (скелетные мышцы и жировая ткань [13].

Это позволяет предположить существование различных циркадианных метаболических фенотипов. Для их определения необходимы обширные контролируемые клинические исследования.

\section{Циркалианные ритмы эн $\triangle$ Окринных прочессов}

Биологические ритмы гормонов являются фундаментом, на котором базируется гомеостаз. Обеспечение биоритмической секреции гормонов поддерживается эфферентными сигналами от СХЯ через нейроэндокринные и вегетативные выходы, которые, в свою очередь, могут давать временные сигналы мишеням в периферические системы и центральную нервную систему (ЦНС). Сбой в биологических ритмах секреции гормонов влечет за собой каскад метаболических изменений и приводит к развитию расстройств и заболеваний, в частности - к ожирению и сахарному диабету 2 типа.

Одним из гормонов, тесно ассоциированным с циркадными ритмами является Тиреотропный гормон (ТТГ). ТТГ секретируется в пульсирующем режиме, обусловленным пульсирующим выбросом тиреотропинрилизинг гормона (ТРГ). При этом частота и амплитуда пульсации увеличиваются в вечернее/ночное время. Пиковый уровень секреции происходит между 23.00 и 04.00 ч, медленно снижается во время сна и в первой половине дня до минимальных значений в послеполуденное время. Также в исследованиях с экспериментальным голоданием обнаружена роль соматостатина 
в регуляции продукции ТТГ: у человека длительное голодание приводит к уменьшению амплитуды пульсовой секреции гормона и пика его концентрации в ночное время, снижению базальной концентрации ТТГ в сыворотке крови [14]. Прием пищи восстанавливает ритм секреции ТТГ, однако, его уровень еще некоторое время остаётся ниже исходного. Интересным представляется влияние продолжительности сна на секрецию ТТГ: как более короткий, так и более длительный сон в сравнении с оптимальной его продолжительностью могут увеличить риск дисфункции щитовидной железы. Так, в исследовании на 5000 пациентов был выявлен повышенный риск субклинического гипертиреоза у короткоспящих (<7 ч/сут) пациентов, который в свою очередь связан с одирением, при отсутствии субклинического гипотиреоза у длинноспящих (>8 ч/сут) людей [15].

Циркадианная система может также играть роль в регуляции аппетита: гормоны, регулирующие аппетит, имеют циркадианный ритм секреции, который сопряжен с циклом «сон-бодрствование». Эндокринные сигналы с периферии оказывают свое воздействие на ежедневный энергетический баланс путем передачи сигналов обратно в мозг циркадианно-зависимо в течение дня. Этими сигналами являются грелин и адипоцитокины, передающие информацию в мозг о наличии или отсутствии периферических запасов или потребностей в энергии и контролирующие массу тела.

Лептин - это адипоцитокин, главным образом секретируемый белой жировой тканью. Транспортируясь в дугообразное ядро гипоталамуса, он ингибирует пул нейронов: нейропептид-Y (NPY- Neuropeptide Y) и агути-подобный белок (AGRP - agouti related peptide), обладающие орексигенным эффектом, и активирует нейроны, секретирующие проопиомеланокортин (РОMC- Proopiomelanocortin) и кокаин-и амфетамин-регулируемый транскрипт (CART- Cocaine and amphetamine regulated transcript), таким образом приводя к снижению потребления пищи. Суточный ритм высвобождения лептина находится под контролем СХЯ. Как правило, уровень лептина увеличивается в ночное время и после еды, что приводит к снижению аппетита. Утром отмечается минимум его секреции. В исследовании при вынужденном смещении суточного ритма у взрослых наблюдались снижение уровня лептина, повышение значений постпрандиальной гликемии, среднего артериального давления, изменение ритма сердца, увеличение секреции инсулина и кортизола с более высокими пиками после пробуждения и перед сном [16].

Таким образом, лептин может представлять собой важную связь между сном, циркадианным ритмом и метаболизмом.
Грелин является гормоном, секретируемым оксинтиновыми клетками слизистой оболочки фундального отдела желудка. Циркулирует он в большей части в неактивной форме и становится биологически активным в ответ на голодание. Грелин противоположен лептину, он вызывает чувство голода, главным образом, через активацию нейронов в дугообразном ядре гипоталамуса, что приводит к повышенному высвобождению NPY и AGRP в паравентрикулярное ядро гипоталамуса (ПВН) и другие гипоталамические структуры. Плазменные уровни грелина колеблются в соответствии с циклом питания, что делает этот гормон предполагаемым кандидатом для регулирования циркадианного пищевого ритма [17]. Повышение общего грелина и снижение активного происходит во время сна, во время бодрствования между приемами пищи в ожидании их наоборот происходит повышение активного грелина. Грелин также подает сигнал обратно в СХЯ, влияя на экспрессию часовых генов, производя сдвиги циркадианного ритма при голоде и ослабляя сигналы в СХЯ в темноте. Следовательно, грелин - эндокринный сигнал, который помогает общаться желудочно-кишечной системе и центральным регуляторам суточного ритма — СХЯ. [18]

Оментин - протеин, который секретируется адипоцитами висцеральной жировой ткани. Он повышает чувствительность к инсулину в мышцах, печени и жировом депо большого сальника. При снижении его секреции увеличивается ИР, что ассоциировано с ожирением и СД 2. В норме пик выработки оментина приходится на дневное время, а к вечеру и в течении ночи его уровень снижается, но при ожирении наблюдается высокая вариабельность его пиков [19]

Уровни лептина, грелина и оментина у пациентов с ожирением выше, чем у пациентов с нормальной ИМТ. Считается, что повышенный уровень с-реактивного белка (СРБ) при ожирении, что приводит к повышению сывороточного уровня лептина [20]. Это сопровождается резистентностью к лептину из-за пониженной регуляции рецепторов к нему и приводит к нарушению регуляции веса и может способствовать его увеличению.

\section{Выво $\triangle \mathrm{b}$}

Циркадианные ритмы являются ключевыми факторами здоровья и благополучия человека. Полученные за последнее десятилетие знания о молекулярных и клеточных основах биологических часов вывели циркадианное время на передний план физиологии и тем самым показали, как временная дезорганизация может лежать в основе заболеваний. Действительно, нарушение суточного ритма, ассоциируются с различными патологиями и риском возникновения расстройств, 
в первую очередь метаболических и эндокринных. Возможно, именно циркадианная дизритмия может объяснить столь быстрый рост ожирения и СД 2. Длительные и тяжелые нарушения сна достаточны для того, чтобы вызвать изменения в гомеостатической энергетической потребности. Прервать «порочный круг» и реализовать лечебный потенциал возможно через хроно- физиологические факторы, в том числе нормализацию сна. Циркадианная концепция должна быть обязательно включена в ядро медицины, чтобы максимально использовать ее потенциал для профилактики и лечения метаболических заболеваний, особенно ожирения и СД 2, что стало крайне актуальным в практике врача-эндокринолога.

\section{ЛИТЕРАТУРА}

1. Hastings M, O'Neill JS, Maywood ES. Circadian clocks: regulators of endocrine and metabolic rhythms. J Endocrinol. 2007; 195: 187-198

2. Circadian Regulation of Glucose, Lipid, and Energy Metabolism in Humans, Eleonora Poggiogalle, Humaira Jamshed, Courtney M. Peterson, Metabolism. in PMC2019 Jul 1, doi: 10.1016/j.metabol.2017.11.017.

3. Panda S. Circadian physiology of metabolism. Science. 2016;354(6315):1008-1015.], [Gerhart-Hines Z, Lazar MA. Circadian metabolism in the light of evolution. Endocr Rev. 2015;36(3):289-304.], [Marcheva B, et al. Circadian clocks and metabolism. Handb Exp Pharmacol. 2013;(217):127-55

4. Takahashi JS. Transcriptional architecture of the mammalian circadian clock. Nat Rev Genet 2017;18:164-79.doi:10.1038/nrg.2016.150],[Eckel-Mahan K, Sassone-Corsi P. Metabolism and the circadian clock converge. Physiol Rev 2013;93:107-35.doi:10.1152/physrev.00016.2012

5. Zhang $\mathrm{EE}$, et al. Cryptochrome mediates circadian regulation of CAMP signaling and hepatic gluconeogenesis. Nat Med. 2010;16(10):1152-6

6. Lee $A$, et al. Diurnal variation in glucose tolerance. Cyclic suppression of insulin action and insulin secretion in normal-weight, but not obese, subjects. Diabetes. 1992;41(6):750-9.

7. Anothaisintawee T, Lertrattananon D, Thamakaison S, et al. Later chronotype is associated with higher hemoglobin A1c in prediabetes patients. Chronobiol Int 2017;34:393-402.doi:10.1080/07420528.2017.1279624

8. Lee $A$, et al. Diurnal variation in glucose tolerance. Cyclic suppression of insulin action and insulin secretion in normal-weight, but not obese, subjects. Diabetes. 1992;41(6):750-9.

9. Leproult R, Holmback U, Van Cauter E. Circadian misalignment augments markers of insulin resistance and inflammation, independently of sleep loss. Diabetbes. 2014;63(6):1860-1869. doi: https://doi.org/10.2337/db13-1546.

10. Van Cauter $\mathrm{EV}$, et al. Abnormal temporal patterns of glucose tolerance in obesity: relationship to sleep-related growth hormone secretion and circadian cortisol rhythmicity. J Clin Endocrinol Metab. 1994;79(6):1797-805

11. Demacker PN, et al. Intra-individual variation of serum cholesterol, triglycerides and high density lipoprotein cholesterol in normal humans. Atherosclerosis. 1982;45(3):259-66.

12. Yoshino J, et al. Diurnal variation in insulin sensitivity of glucose metabolism is associated with diurnal variations in whole-body and cellular fatty acid metabolism in metabolically normal women. J Clin Endocrinol Metab. 2014;99(9): E1666-70.

13. Circadian Regulation of Glucose, Lipid, and Energy Metabolism in Humans, Eleonora Poggiogalle, Humaira Jamshed, Courtney M. Peterson, Metabolism. in PMC2019 Jul 1, doi: 10.1016/j.metabol.2017.11.017.

14. Romijn J.A., Adriaanse R., Brabant G., Prank K., Endert E., Wiersinga W.M. Pulsatile secretion of thyrotropin during fasting: a decrease of thyrotropin pulse amplitude. // J Clin Endocrinol Metab. — 1990 — Vol. 70. — P. 1631-1636

15. Woojun Kim 1, Jeongmin Lee, Jeonghoon Ha, Kwanhoon Jo, Dong-Jun Lim, Jung-Min Lee, Sang-Ah Chang, Moo-Il Kang, Min-Hee Kim, Association between Sleep Duration and Subclinical Thyroid Dysfunction Based on Nationally Representative Data, Journal of Clinical Medicine 2019, 8(11), 2010; https://doi. org/10.3390/jcm8112010.

16. Scheer FA, Hilton MF, Mantzoros CS, Shea SA. Adverse metabolic and cardiovascular consequences of circadian misalignment. Proc Natl Acad Sci U S A. 2009;106(11): 4453-4458

17. Patton, D.F., Mistlberger, R.E., 2013. Circadian adaptations to meal timing: neuro- endocrine mechanisms. Front. Neurosci 7, 185

18. Spiegel K, et al. Twenty-four-hour profiles of acylated and total ghrelin: relationship with glucose levels and impact of time of day and sleep. J Clin Endocrinol Metab. 2011;96(2):486-93

19. S. Zirlik1, K.M. Hildner1, A. Targosz3, M.F. Neurath1, F.S. Fuchs1, T. Brzozowski3, P.C. Konturek. Melatonin And Omentin: Influence Factors In The Obstructive Sleep Apnoea Synd Rome?, Journal Of Physiology And Pharmacology 2013, 64, 3, 353-360

20. Chen K, Li F, Li J, et al. Induction of leptin resistance through direct interaction of C-reactive protein with leptin. Nature Medicine. 2006;12(4):425-432

( Венгржиновская Оксана Игоревна ( vengrzhinovskay@gmail.com ), Фадеева Мария Игоревна. Журнал «Современная наука: актуальные проблемы теории и практики» 